Jahrbuch Schweiz - Dritte Welt 1995

\title{
Für eine kohärentere schweizerische Politik gegenüber den Ländern des Südens
}

Einige kritische Betrachtungen anhand des Falls Indonesien

Jean-Luc Maurer

\section{(2) OpenEdition}

\section{Journals}

Electronic version

URL: http://journals.openedition.org/sjep/1300

DOI: $10.4000 /$ sjep.1300

ISSN: 1663-9677

\section{Publisher}

Institut de hautes études internationales et du développement

Printed version

Date of publication: 1 février 1995

Number of pages: 167-177

ISSN: 1660-5926

\section{Electronic reference}

Jean-Luc Maurer, «Für eine kohärentere schweizerische Politik gegenüber den Ländern des Südens », Schweizerisches Jahrbuch für Entwicklungspolitik [Online], 14 | 1995, Online erschienen am: 05 Mai 2013, abgerufen am 08 September 2020. URL : http://journals.openedition.org/sjep/1300 ; DOI : https://doi.org/10.4000/sjep.1300 


\title{
Für eine kohärentere schweizerische Politik gegenüber den Ländern des Südens
}

\author{
Einige kritische Betrachtungen anhand \\ des Falls Indonesien
}

Jean-Luc Maurer, Asienspezialist, IUED

Indonesien ist eine ausgezeichnete Fallstudie, um Betrachtungen über die Kohärenz der Aussenpolitik der Schweiz gegenüber den Ländern des Südens anzustellen. Zuerst einmal ist Indonesien - nach China und Indien - das drittgrösste Entwicklungsland der Erde und das einzige in Südostasien, wo der Bund seit Anfang der 70er Jahre beschlossen hat, seine öffentliche Hilfe auf dieses Land zu konzentrieren. Ferner ist es ein Land, das noch lange mit schwerwiegenden Entwicklungsproblemen zu kämpfen haben wird, das aber auch als bedeutsamer Wirtschafts- und Handelspartner auf der regionalen und internationalen Bühne aufzutreten beginnt, ein Land, in dem die schweizerischen Privatinteressen seit langem bestehen und nicht unerheblich sind. Schliesslich ist es ein Land, dessen Entwicklungspolitik bemerkenswerte wirtschaftliche und soziale Ergebnisse hervorgebracht hat, das aber auch durch ein autoritäres Regime gekennzeichnet ist, das eine sehr merkwürdige Konzeption der Achtung der Menschenrechte hat und wo der Druck auf die natürlichen Ressourcen grosse Umweltprobleme aufwirft. Kurz gesagt, Indonesien ist somit a priori ein idealer Partner, um eine kohärentere Aussenpolitik zu definieren und $z u$ versuchen, diese umzusetzen. Bevor wir untersuchen, welches die Hauptziele einer solchen Politik sein sollten und welches ihr zentraler Mechanismus sein könnte, seien die vier Ebenen analysiert, auf denen sich Kohärenzwidersprüche ergeben können.

\section{Vier mögliche Analyseebenen der Beziehungen Schweiz-Indonesien}

\section{Entwicklungszusammenarbeit und wirtschaftliche Entwicklung}

Die Schweiz hat sich seit Anfang der 70er Jahre sehr aktiv in der Entwicklung Indonesiens eingesetzt, als die neue Regierung, welche aus den blutigen politischen Ereignissen von 1965-66 hervorgegangen war, an die westliche Hilfe appellierte, um den wirtschaftlichen Wiederaufstieg des Archipels zu finanzieren, und Indonesien zum Schwerpunktland der DEH in Südostasien wurde. Neben der Tatsache, dass die Schweiz Mitglied des internationalen Konsortiums für Indonesien ist (IGGI jetzt CGI) und jedes Jahr zur multilateralen Finanzierung seiner Entwicklung beiträgt, hat sie in 25 Jahren ein bilaterales Pro- 
gramm der Entwicklungszusammenarbeit durchgeführt, das zwar bescheiden, aber gut konzipiert ist und von unserem Partner wegen seines Geistes und seiner Qualität geschätzt wird. Dieses Programm hat das Schwergewicht auf die technische Berufsbildung und die Verbesserung der Lebensbedingungen der ärmeren Bevölkerungsgruppen gelegt, und zwar in Java eher im städtischen Bereich und auf den anderen Inseln weitgehend im ländlichen Bereich.

Ohne hier auf Einzelheiten einzugehen ${ }^{1}$, hat Indonesien in diesem Vierteljahrhundert bei seiner Entwicklung enorme Fortschritte gemacht. Dieses Land, das zu Beginn der 60er Jahre von einigen als der offensichtlichste Fall von Unterentwicklung auf der Welt angesehen wurde, erscheint heute vielen als eines der nächsten „neuen Industrieländer“ Asiens. Dieser überwältigende Erfolg ist zuerst einmal das Ergebnis einer pragmatischen und sinnvollen Entwicklungspolitik der indonesischen Regierung, die es verstanden hat, die richtigen Prioritäten zu setzen und die notwendigen Investitionen vorzunehmen. Jedoch verdankt dieser Erfolg auch viel der Unterstützung, die diese Politik von aussen her seitens der Weltbank, Japans und der westlichen Länder erhalten hat, welche im IGGI zusammengeschlossen sind. In diesem Rahmen ist die Rolle der Schweiz, obwohl sie beschränkt war, nicht unerheblich gewesen. Mit anderen Worten haben wir zur Verwirklichung einer „erfolgreichen Entwicklungsgeschichte" in einem grossen Land des Südens beigetragen. Der Fall ist selten genug seit Beginn der Unabhängigkeit der Länder der Dritten Welt, um nachdrücklich hervorgehoben zu werden. Man sollte sich übrigens zu diesem Erfolg offen beglückwünschen und ihn in viel grösserem Umfang bei der öffentlichen Meinung bekanntmachen, während die Medien eher Tendenz haben, diese bezüglich der Fehlschläge der internationalen Entwicklungszusammenarbeit in Afrika zu sensibilisieren.

Im Laufe dieses Entwicklungsprozesses hat sich das indonesische Bruttosozialprodukt pro Einwohner um mehr als das Elffache erhöht, wobei es von 60 US Dollar im Jahre 1969 auf 670 US Dollar 1992 anstieg. Der Sprung nach vorne ist zwar eindrucksvoll, doch ist das Ergebnis noch recht bescheiden, da Indonesien gemäss der Weltbank gerade an der Grenze zwischen den Volkswirtschaften mit niedrigem Einkommen und denjenigen mit mittlerem Einkommen liegt, auf dem gleichen Niveau wie Länder, von denen man a priori denken könnte, dass sie viel weniger entwickelt sind als Indonesien, wie Ägypten, die Elfenbeinküste oder Bolivien, und zum Beispiel weit unter dem Niveau Perus, das 1992 praktisch bereits die 1000 US Dollar-Grenze erreichte. Gleichwohl hat die DEH gerade unter Berufung auf die Tatsache, dass Indonesien sich seit zwanzig Jahren stark entwickelt habe und nicht mehr den Haupt-

1. Siehe zu diesem Thema den Beitrag des Autors über die wirtschaftliche, soziale und politische Entwicklung Indonesiens im Werk von G. Etienne, J.L. Maurer und C. Renaudin, Suisse-Asie, pour un nouveau partenariat, Genf, Verlag Olizane und CRAM (IUHEI/IUED), 1992, S. 83-128 
kriterien des schweizerischen Entwicklungshilfegesetzes von 1976 entspreche - welche festlegen, dass unsere öffentliche Entwicklungshilfe sich auf die ärmsten Länder, Gebiete oder Bevölkerungsgruppen konzentrieren soll - beschlossen, sich bis zum Jahre 2000 aus diesem Lande zurückzuziehen. Jedoch leben, den zuverlässigsten Quellen zufolge, noch 15 bis 20 Prozent der indonesischen Bevölkerung, das heisst 30 bis 35 Millionen Menschen unter der absoluten Armutsgrenze, die meisten von innen auf Java, der am weitesten entwickelten, aber auch am stärksten bevölkerten Insel des Archipels. Diese Zahl allein spricht für sich selbst.

Von dieser ganz einfachen Analyse ausgehend, und da dieses Schwerpunktthema des Jahrbuchs Schweiz-Dritte Welt den Zweck hat, sich über die Kohärenz unserer Aussenpolitik Gedanken zu machen, sei es uns erlaubt, hier zu sagen, dass uns dieser Rückzugsbeschluss nicht die beste Demonstration hierfür gewesen zu sein scheint. Er wurde im übrigen ohne grosse Konsultation und ohne Debatte, fast heimlich, mit einer etwas beschämenden Hast getroffen. Das ist sehr schade, denn wenn die Reflektion über die Kohärenz, die wir in Bezug auf Indonesien seit Jahren befürworten, in Absprache erfolgt wäre, hätte sie vielleicht zu einer anderen Entscheidung geführt, die heute eine grössere Auswahl an Möglichkeiten für unsere Aussenpolitik gegenüber dieser bedeutenden Regionalmacht Asiens bieten würde. Auch wenn der Beschluss, sich zurückzuziehen, letztlich die Oberhand gewonnen hätte, so hätte die Übertragung der Aufgaben an andere nationale Partner zudem unter besseren Bedingungen erfolgen können als denen, die Aufgaben fallen zu lassen, indem man hofft, dass sie von anderen übernommen werden. Schliesslich wäre es, selbst wenn ein solcher Konsultationsprozess zu einem Rückzugsbeschluss geführt hätte, möglich gewesen, der Schweizer Öffentlichkeit klar und deutlich mitzuteilen, dass die DEH sich aus Indonesien wegen eines überzeugenden Erfolgs im Entwicklungsbereich zurückziehe und in diesem Stadium die Aufgaben anderen nationalen Akteuren überlasse, die direkter betroffen sind.

\section{Entwicklungszusammenarbeit und Aussenwirtschaftsbeziehungen}

Seit zwanzig Jahren ist Indonesien im Laufe seiner Entwicklung auch zu einem immer bedeutenderen Handels- und Wirtschaftspartner für die Schweiz geworden. Zwar haben sich die multinationalen Schweizer Grosskonzerne wie Nestlé, Ciba Geigy, Diethelm oder die SGS bereits seit langem in Indonesien niedergelassen, da sie sich des enormen Potentials der indonesischen Wirtschaft und eines Binnenmarktes von bald 200 Millionen Einwohnern bewusst sind. Jedoch haben sich der Handelsaustausch und die Investitionen zwischen den beiden Ländern vor allem seit der wirtschaftlichen Liberalisierung der letzten zehn Jahre erhöht. Indonesien erreicht ein Stadium seiner industriellen Entwicklung, in dem es die technologischen Stufen durchlaufen muss, die vor inm Südkorea, Taiwan, Hongkong, Singapur oder Thailand und Malaysia 
durchlaufen haben. In diesem Stadium sollte das Know-How zahlreicher Schweizer Klein- und Mittelindustrien Indonesien am nützlichsten sein. Unsere Dienstleistungsunternehmen haben in dieser Hinsicht ebenfalls eine ziemlich gute Ausgangsposition, sowohl im Banken- und Versicherungsbereich als auch im Hotelgewerbe und im Tourismus. Die wirtschaftlichen Aussichten sind somit von vornherein eher gut, sofern diese Unternehmen bereit sind, Risiken einzugehen. Leider scheint es, dass dies wieder eine ganz andere Frage ist. Jedenfalls haben die Geschäftskreise der meisten anderen Industrieländer wohl begriffen, was hier auf dem Spiel steht, und sind im Durchschnitt aktiver als unsere Vertreter auf diesem aussichtsreichen Markt.

Andererseits wird Indonesien noch lange ein Entwicklungsland, mit seinen klassischen wirtschaftlichen und sozialen Problemen bleiben. Deshalb wird es noch während eines guten Teils der ersten Hälfte des nächsten Jahrhunderts die öffentliche Entwicklungshilfe Japans, seinem ersten Wirtschaftspartner und Geldgeber, erhalten können, sowie die Hilfe der meisten grossen westlichen Länder, die in Indonesien stark engagiert sind, wie Deutschland, Australien oder die USA. Die Mehrheit der westlichen Staaten werden somit gegenüber Indonesien weiterhin eine Aussenpolitik betreiben, die dem „dualistischen“ Charakter dieses Landes entspricht, das gleichzeitig ein Empfänger von Ressourcen der Entwicklungszusammenarbeit und ein vollwertiger Wirtschafts- und Handelspartner ist. Dies ist a priori die einzige sinnvolle Politik gegenüber solchen Entwicklungsländern, die sich in einer Übergangsphase zwischen einer noch weitgehend traditionellen Wirtschaft und der industriellen Konsumgesellschaft befinden.

Das Problem ist, dass unser eidgenössisches Verwaltungssystem für eine solche Politik wenig vorteilhaft ist. Die Entwicklungszusammenarbeit untersteht der Zuständigkeit des EDA und der DEH, während die Wirtschafts- und Handelsbeziehungen in den Zuständigkeitsbereich des EVD und des BAWI fallen. Hieraus ergibt sich: wenn ein Land arm ist, wie Nepal, dann hat die DEH die Ausschliesslichkeit dafür, oder wenn es wohlhabender geworden ist, wie Südkorea, dann fällt es in den Kompetenzbereich des BAWI. Kurz gesagt, wir haben hier vielmehr das typisch helvetische System des "entweder - oder" statt einer feineren und nuancierteren Politik, wie sie die meisten Situationen erfordern würden. Nun muss aber zwischen der Maxime „trade not aid“ der Anhänger des Ultraliberalismus und der Devise „aid without trade“ einiger Integristen der Entwicklungszusammenarbeit ein goldener Mittelweg gefunden werden, insbesondere für solche Länder, die sich in der Übergangsphase befinden. Was vielleicht für den Niger oder Nepal gilt, ist jedenfalls keineswegs gültig für Indonesien und wahrscheinlich auch bald nicht mehr für Indien und Pakistan, die beiden wichtigsten Schwerpunktländer der DEH auf dem indischen Subkontinent.

Im übrigen ist die Zusammenarbeit zwischen DEH und BAWI niemals sehr eng und auch nie wirklich gut gewesen. Zwischen den Beamten der beiden Verwaltungen gibt es mehr Rivalität und Misstrauen als den Willen, an der Ausarbeitung einer abgestimmten kohärenten Politik mitzuwirken. Hier liegt un- 
ser grösster Widerspruch. Hat man wirklich den Willen und die Fähigkeit, eine kohärente Aussenwirtschaftspolitik mit den Ländern des Südens auszuarbeiten - insbesondere mit den Ländern des "dualistischen“ Typs wie Indonesien - die es erlauben würde, weiterhin Aktivitäten im Bereich der öffentlichen Entwicklungszusammenarbeit durchzuführen und gleichzeitig den Umfang der Handelsbeziehungen und der privaten Investitionen zu steigern? Wie kann man jetzt - wo die Entscheidung getroffen wurde, das Entwicklungszusammenarbeitsprogramm in Indonesien abzubrechen - vorgehen, um wiedergutzumachen, was diesbezüglich noch möglich ist? Der sehr rasche Rhythmus des Rückzugs erleichtert jedenfalls die Dinge nicht. Ohne zu sehr auf diesen Punkt eingehen zu wollen, scheint es uns, dass man hier trotzdem bereits weitgehend die Gelegenheit verpasst hat, gegenüber einem Land des Südens eine wirklich kohärente Politik zu erstellen, während der Fall Indonesiens wiederum ideal gewesen wäre, um dies zu tun.

\section{Wirtschaftsentwicklung und Demokratisierung des politischen Systems}

Ausser einer zu raschen Entwicklung, die für Indonesien paradoxerweise Probleme wie die obenerwähnten mit sich bringt, ist die andere grosse Schwierigkeit, vor die sich Indonesien in der Schweiz und in vielen anderen westlichen Ländern immer gestellt sah, mit dem autoritären und repressiven Charakter des militärisch-technokratischen Regimes verbunden, das aus den schrecklichen blutigen Ereignissen von 1965-66 hervorgegangen ist. Wenn das Regime das Land auch zweifellos aus dem wirtschaftlichen Elend und der Verarmungsspirale herausgezogen hat, in die es versunken war, so ist die Neue Ordnung General Suhartos, der seither in Djakarta regiert, in der Tat weit davon entfernt, auch nur die elementarsten Regeln der Demokratie einzuhalten.

Das schwerwiegendste Problem hängt mit der Annektion Osttimors im Jahre 1975 zusammen und mit der politischen Unterdrückung, die dort seitdem herrscht, trotz der löblichen wirtschaftlichen Entwicklungsanstrengungen, die dort unternommen werden. Dies ist jedoch kein Einzelfall und man trifft anderswo im Archipel Probleme systematischer Unterdrückung des Selbstbestimmungsrechts der Völker an. Zudem wird die freie Ausübung der gewerkschaftlichen Rechte noch weitgehend mit Füssen getreten: die offizielle Gewerkschaft steht unter dem Joch der Regierung, und Anwandlungen autonomer, weniger willfähriger Meinungsäusserungen werden gewaltsam unterdrückt, wie einige höchst tragische Ereignisse zeigen, die sich vor kurzen zugetragen haben. Schliesslich werden die freie Meinungsäusserung und die Pressefreiheit streng überwacht, was die unaufhörlichen Probleme beweisen, denen sich die Stundentenverbände ausgesetzt sehen, wie auch die kürzliche Schliessung von drei der grössten nationalen Wochenzeitungen. Kurz gesagt ist die indonesische Neue Ordnung eine gelenkte Teildemokratie, die den Anschein der Wahlen alle fünf Jahre rituell aufrechterhält, die aber mit wahrhaft eiserner Hand geführt wird, auch wenn der Handschuh weiterhin aus Samt ist. 
Obwohl diese Aspekte beim Beschluss der DEH, ihr Programm vor dem Jahre 2000 einzustellen, scheinbar keine förmliche Rolle gespielt haben, steht fest, dass das Image Indonesiens bei der öffentlichen Meinung in den westlichen Ländern niemals gut war, vor allem nach den blutigen Ereignissen, die Ende 1991 in Timor stattfanden. Das ist gut zu verstehen. Kann man jedoch das Problem nicht auch unter einem anderen Blickwinkel betrachten? Unseres Wissens nach ist niemals und nirgendwo die Demokratie der Entwicklung vorangegangen. Ohne bis aufs Alterum zurückzugehen, haben sich die Völker erst einmal darum gekümmert „einen vollen Bauch“ zu haben, bevor sie weniger materielle Freiheiten forderten. Die europäische Geschichte seit dem Mittelalter beweist dies reichlich. In jüngerer Zeit haben auch autoritäre und eher repressive Regimes, die demjenigen Indonesiens unter General Suharto sehr ähnlich sind, die spektakuläre Entwicklung Südkoreas oder Taiwans ermöglicht. Dieser Entwicklungsprozess hat zum Auftreten neuer sozialer Akteure geführt, nämlich einer Arbeiterklasse, die sich ihrer Macht immer mehr bewusst ist und sich organisiert hat, sowie einer Mittelklasse, die es nicht mehr hinnimmt, von der politischen Macht ferngehalten zu werden. Seit einigen Jahren haben diese autoritären Regimes damit begonnen, sich zu demokratisieren und sehen sich vor wachsende Forderungen nach mehr individuellen und kollektiven Freiheiten gestellt.

Tatsächlich sind autoritäre Regimes, welche die Entwicklung eines Landes fördern, stets Opfer ihres eigenen Erfolgs. Indonesien bildet da keine Ausnahme, jedoch mit der zeitlichen Verschiebung, die mit ihrem niedrigeren Entwicklungsstand verbunden ist. Die Arbeiterklasse hat damit begonnen, sich zu organisieren, und die Mittelklasse beginnt Forderungen zu stellen. Man muss auch zur Entlastung Indonesiens, dem grössten Moslemstaat der Welt, sagen, dass dort eine Glaubensfreiheit, eine Achtung vor der kulturellen Vielfalt und eine allgemeine Toleranz herrschen, die immer seltener werden, in den Entwicklungsländern des Südens wie auch in den Ländern des Nordens, von denen man annahm, sie seien entwickelt. Ist es in diesem Zusammenhang notwendig, an den Fall Jugoslawiens zu erinnern? Die Fortsetzung der Entwicklung kann nur zur Beschleunigung dieses Prozesses beitragen. Wäre es somit nicht besser, anstatt der indonesischen Regierung eine heuchlerische jüdisch-christlich inspirierte Moral über Menschenrechte und Demokratie entgegenzusetzen eine weitgehend unangebrachte Haltung in Anbetracht der fragwürdigen Leistungen des Westens in diesem Bereich, die aufgrund der zur Kolonialzeit erlebten traumatisierenden geschichtlichen Erfahrung sehr schlecht aufgenommen wird - einen Entwicklungsprozess zu unterstützen, der alle Aussichten hat, rasch die erwarteten sozialen und politischen Auswirkungen zu erzielen?

Zum anderen kann man sich auch sagen, dass man nicht am meisten Chancen hat, auf die politischen Ausrichtungen einer Regierung Einfluss nehmen zu können, indem man sich zurückzieht und zurückhält. Im Gegenteil, nur indem man sich mehr bei der Entwicklung einsetzt und wachsende Anforderungen als vollwertiger Partner stellt, kann man den Dialog vertiefen. Letzterer kann in der Tat nur auf Vertrauensbeziehungen beruhen. Aber Vertrauen 
lässt sich nicht an einem Tage gewinnen. Die ganze Energie, die man seit 25 Jahren in Indonesien aufgebracht hat, um eine solche Beziehung herzustellen, um dieses komplexe Land besser zu kennen und zu lernen, mit einem schwierigen Partner zu arbeiten, wird durch die Entscheidung, sich so überstürzt zurückzuziehen, erheblich belastet. Wir müssen jetzt wieder von vorne anfangen mit einem anderen Schwerpunktland in Südostasien, mit Vietnam, wo übrigens fast alle zur gleichen Zeit eintreffen. Zwar handelt es sich um ein Land, dessen Bedürfnisse enorm sind, bei dem aber der Stand der in der Schweiz angehäuften Kenntnisse noch sehr niedrig ist, wo die wirtschaftlichen und sozialen Probleme ungefähr gleicher Art sind wie in Indonesien, wo das politische Regime mindestens genau so autoritär ist, wo die Korruption kaum weniger verbreitet ist und wo die Menschenrechtslage auf jeden Fall nicht besser ist.

\section{Rasche wirtschaftliche Entwicklung und Umweltschäden}

Wenn man in Betracht zieht, dass Umweltprobleme weitgehend eine Folge der Übervölkerung sind, muss man ebenfalls anerkennen, dass die indonesische Regierung sich in diesem Bereich grosse Verdienste erworben hat. Die Politik der Geburtenbeschränkung, die sie seit rund zwanzig Jahren eingeführt hat, hat in der Tat sehr ermutigende Ergebnisse gezeigt, da die natürliche Wachstumsrate unter die $2 \%$-Grenze gefallen ist. Hingegen gibt es zahlreiche andere Umweltprobleme. Das schwerwiegendste ist das rasch voranschreitende Abholzen der Tropenwälder auf Borneo und auf anderen Inseln. Aber auch hier liegt die Lösung nicht in einer Moralpredigt über die rationelle Bewirtschaftung der Forstressourcen als gemeinsamem Erbe der Menschheit. Dem Westen steht es schlecht an, in dieser Beziehung Moral zu predigen, nachdem er die Erde verwüstet hat und die eingeborenen Völker ausgerottet hat, wo immer er sich hin begeben hat, um seine Herrschaft auszuüben. Die Lösung liegt eher in der Unterstützung bei der Heranbildung eines indonesischen Umweltbewusstseins, das angesichts einer Entwicklung aufkommt, die zu sehr auf sofortigen wirtschaftlichen Gewinn und rein materielle Werte ausgerichtet ist. Andere wesentliche Umweltprobleme ergeben sich bei der Wasserbewirtschaftung, beim übermässigen Einsatz von Pestiziden, beim Bergbau oder bei der Hochseefischerei. Man könnte jedoch nicht besser zu ihrer Lösung beitragen, als die lokalen NGO zu fördern, die in diesem Sektor am aktivsten sind.

\section{Prioritätensetzung bei den Zielen und Streben nach besserer Kohärenz}

\section{Hauptziel: den indonesischen Entwicklungsprozess fördern}

Das Globalziel der schweizerischen Aussenpolitik gegenüber Indonesien sollte logischerweise sein, dass der Lebensstandard der Bevölkerung sich weiter erhöht, dass die Demokratisierung des politischen Systems voranschreitet und 
dass die Umweltprobleme ihre Lösungen finden. Hierzu muss das Land seine Entwicklung fortsetzen, indem es seine Wirtschaft diversifiziert und seine Industrialisierung beschleunigt, um Arbeitsplätze zu schaffen und die enormen Massen von Jugendlichen aufnehmen zu können, die jedes Jahr auf den Arbeitsmarkt gelangen. Zu diesem Zweck muss das Land eine wachsende Anzahl qualifizierter junger Indonesier für den Arbeitsmarkt ausbilden. Das bedeutet, dass die Bereiche Erziehung und Berufsausbildung - in denen sich die schweizerische Entwicklungszusammenarbeit seit 25 Jahren stark eingesetzt hat prioritär bleiben. Nun ist in diesem Stadium die Fortsetzung einer externen Förderung im Rahmen der öffentlichen Entwicklungshilfe wahrscheinlich weiterhin notwendig, damit die in Angriff genommenen wichtigen Reformen wirklich zu Ende gebracht werden können. Dies hat ein Land wie Deutschland verstanden und beschlossen, sein ganzes Gewicht und seine lange Erfahrung in diesem Bereich in die Waagschale zu werfen.

Somit bleibt die Priorität bei der Verbesserung der wirtschaftlichen Lage, eine Grundbedingung, um das Bevölkerungswachstum weiterhin zurückgehen zu lassen, den allgemeinen Lebensstandard der Bevölkerung zu heben und die Einkommensunterschiede zwischen den sozialen Schichten zu reduzieren, um das Aufkommen neuer gesellschaftlicher Akteure zu fördern, die einen wachsenden Druck zugunsten einer stärkeren Demokratisierung des politischen Systems ausüben werden. Kurzum, man muss diesem Land weiterhin helfen, sich wirtschaftlich zu entwickeln, weil dies das einzige Mittel ist, um für die anderen oben angesprochenen Probleme im sozialen und politischen Bereich allmählich eine Lösung zu finden.

Folglich wäre es im Hinblick auf die Kohärenz der Schweizer Aussenpolitik logisch, dass die Schweiz die wirtschaftliche Entwicklung Indonesiens weiterhin unterstützt, sowohl durch ihre öffentliche Hilfe als auch durch die Intensivierung ihres Handelsaustausches mit diesem Land, sowie ihrer privaten Investitionen vor Ort. Dies würde im Idealfall bedeuten, dass der Beschluss, das Programm der Entwicklungszusammenarbeit mit Indonesien bis zum Jahre 2000 einzustellen, überprüft wird und dass der vorgesehene Rhythmus des Rückzugs zumindest verlangsamt wird. Dann wäre es sicherlich leichter, Mechanismen der Zusammenarbeit einzusetzen und zu erproben, die gewährleisten, dass die DEH die Aufgaben der bilateralen Wirtschaftsbeziehungen anderen nationalen staatlichen Akteuren wie dem BAWI oder der Schweizerischen Zentrale für Handelsförderung und privaten Akteuren wie der neuen Handelskammer Schweiz-Südostasien oder den Berufsverbänden der betroffenen Industriebranchen, sowie den nichtstaatlichen oder kirchlichen Organisationen übertragen kann, die sich weiterhin direkt an der wirtschaftlichen und sozialen Entwicklung Indonesiens beteiligen wollen. Doch können die sehr eng aufeinanderfolgenden Termine, die für den Rückzug festgesetzt wurden, auch einen positiven Faktor darstellen, indem sie die betroffenen Akteure zwingen, rasch unter Zwang zu handeln. Wie dem auch sei, all das setzt auch voraus, dass die schweizerischen privaten Kreise, insbesondere die kleinen und mittleren Unternehmen, parallel hierzu aus ihrer Zurückhaltung heraustreten und sich aktiver 
für das enorme Potential dieses ausgedehnten Landes interessieren.

Andererseits sollte dies nicht die Entscheidung der DEH in Frage stellen, nach und nach ein Programm der Entwicklungszusammenarbeit mit Vietnam als neuem Schwerpunktland in Südostasien zu entwickeln. Tatsächlich könnte ein allmählicher und erheblich langsamer als vorgesehen erfolgender Rückzug aus Indonesien mit der Steigerung des Einsatzes in Vietnam gekoppelt werden. Es braucht gute zehn bis fünfzehn Jahre, um mit Vietnam ein Programm der Entwicklungszusammenarbeit zu erstellen, das jenem vergleichbar ist, das wir mit Indonesien aufgebaut haben. Zuerst einmal müsste das Nötige getan werden, um in der Schweiz einen Pool an Kompetenzen zu bilden, der vergleichbar ist mit demjenigen, der uns über Indonesien zur Verfügung steht. Ein solcher Pool ist unerlässlich, um in diesem neuen Land wirksam zu arbeiten. Im übrigen, wenn Vietnam sich in dem Rhythmus entwickelt, wie es behauptet, ist es nicht unmöglich, dass es einen Entwicklungsstand erreicht haben wird, der mit dem Niveau des heutigen Indonesiens vergleichbar ist, bevor das Programm der DEH dort wirklich bedeutsam ist. Deshalb müsste man von vorherein die Koordinationsmechanismen auf nationaler Ebene einsetzen, die es erlauben, sich unter besseren Bedingungen aus Vietnam zurückzuziehen, als das eventuell in Indonesien der Fall sein wird, wenn Vietnam seinerseits - vielleicht rascher als vorgesehen - einen Entwicklungsstand erreicht haben wird, bei dem es für die öffentliche Entwicklungshilfe der Schweiz nicht mehr in Frage kommt.

\section{Kohärenz durch die Dreieckskoordination fördern}

Es ist sicher, dass das Entwicklungshilfegesetz von 1976 von vornherein nicht sehr gut geeignet ist für die Ausarbeitung kohärenter Aussenpolitiken gegenüber Entwicklungsländern, die sich in einem wirtschaftlichen Übergangsstadium befinden, wie Indonesien. Jedoch muss im gegenwärtigen Kontext vermieden werden, das Gesetz in Frage zu stellen und sogar es abzuändern, denn die Gefahr ist zu gross, „die Büchse der Pandora zu öffnen“. Hingegen ist es unerlässlich, die Kriterien auszuarbeiten, die in Bezug auf die Anwendungsmodalitäten des Gesetzes eine viel flexiblere Auslegung je nach Art des betreffenden Entwicklungslandes ermöglichen.

Grundlegender scheint es uns, dass das Fundament jeder künftigen kohärenten Politik mit Indonesien oder mit jedem anderen Entwicklungsland, das der gleichen Kategorie angehört, die Einsetzung von Mechanismen der Koordination und Zusammenarbeit zwischen der öffentlichen Verwaltung, der Privatwirtschaft und den Akademikerkreisen sein muss. Im Augenblick ist die Lage in der Schweiz in dieser Hinsicht besonders ungesund. In der Tat arbeitet die öffentliche Verwaltung in ihrem Bereich, ohne viel Beziehungen zum Privatsektor und zu den Universitäten zu unterhalten; der Privatsektor blickt gewöhnlich auf die öffentliche Verwaltung und die Akademikerkreise herab, während die Akademikerkreise einerseits frustriert sind, nicht konsultiert zu werden, und 
andererseits weitgehend unfähig sind, aus ihrem Elfenbeinturm herauszutreten und sich auch nur im geringsten ernsthaft in Frage zu stellen. Angesichts dieser Lage geht es effektiv darum, zwischen diesen drei Polen die berühmte „Dreiecksbeziehung“ herzustellen, wie das einige Länder bereits seit langem tun, Japan in meisterhafter und unvergleichlicher Art, andere Länder wie die USA und Deutschland auf jeden Fall besser als die Schweiz.

Bevor die Koordination zwischen den drei betroffenen Partnern erfolgen kann, muss jeder von ihnen sich erst einmal verstärken. Auf Seiten der öffentlichen Verwaltung muss eine wesentliche Arbeit zwischen der DEH und dem BAWI geleistet werden, die sich besser kennenlernen müssen und lernen müssen, zusammenzuarbeiten. Andere Bundesämter sind ebenfalls betroffen. Auf Seiten der Privatindustrie müssen die grossen Unternehmen ihre Beziehungen zu den kleinen und mittleren Unternehmen in jedem Tätigkeitsbereich intensivieren und ihnen gegenüber eine Rolle der Schirmherrschaft übernehmen. Ferner ist es auch sehr wichtig, dass die Arbeitgeber die Gewerkschaften bei der Ausarbeitung der Industriepolitiken gegenüber den Ländern des Südens stärker heranziehen. Schliesslich müssen die NGO lernen, zusammenzuarbeiten, anstatt sich gegenseitig zu ignorieren oder in ständiger Konkurrenz zueinander zu stehen. Auf Seiten der akademischen Kreise sollten die verschiedenen „centres d'excellence" des Landes ebenfalls lernen, sich gegenseitig zu ergänzen und ihre Erfahrungen auszutauschen. Nachdem dies geschehen ist, sollten die drei Pole des Dreiecks untereinander Beziehungen errichten und diese ausbauen. So muss sich die öffentliche Verwaltung daran gewöhnen, enger mit den Kreisen der Privatindustrie zusamenzuarbeiten, und diese müssen sich daran gewöhnen, die Verwaltung etwas weniger herabzusetzen, während die Akademikerkreise lernen müssen, mit diesen beiden Partnern besser zusammenzuarbeiten, indem sie mit innen umfassendere Beziehungen entwikkeln. All dies setzt somit voraus, dass die drei betroffenen Akteure bereit sind, sich in Frage zu stellen, und den Geist der Versöhnlichkeit walten lassen.

Der obenerwähnte Dreiecksprozess ist komplex und erfordert Zeit. Um unverzüglich voranzukommen, ist das beste, durch die Praxis zu lernen. Der Fall Indonesiens eignet sich gut für eine solche Übung. Man könnte sich beispielsweise eine erste Dreiecksübung mittels der Einsetzung einer nationalen dreigliedrigen Kommission über Indonesien vorstellen. Die Kommission wäre damit beauftragt, alle Bestandteile der Politik der drei Partner zu untersuchen, deren Koordination zu gewährleisten und deren Hauptwidersprüche zu beseitigen, um ein integriertes und harmonisches Programm auszuarbeiten, das es der Schweiz gestattet, gegenüber Indonesien eine kohärente Aussenpolitik zu führen. Die gleiche Übung müsst für alle grossen Entwicklungsländer vergleichbarer Art erfolgen, mit denen die Schweiz Beziehungen der Entwicklungszusammenarbeit unterhält, Handel treibt und im Universitätsbereich zusammenarbeitet. Neben Indonesien denkt man hier vor allem an China, Indien, Pakistan und Vietnam in Asien, an Brasilien, Mexiko, Chile, Argentinien und Peru in Lateinamerika, sowie an Südafrika. Wenn jede dieser nationalen dreigliedrigen Kommissionen funktioniert und ein Minimum an Erfahrung er- 
langt hat, kann man daran denken, eine gemischte dreigliedrige Kommission für jedes Land zu errichten, die auch Vertreter der drei Partner aus dem Land des Südens umfasst.

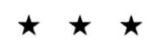

Fangen wir aber am Anfang an, nämlich bei Indonesien. Der Augenblick hierzu ist gut gewählt, und vielleicht können wir somit eine kohärentere Aussenpolitik ausarbeiten, mittels derer die DEH bis Ende des Jahrhunderts ihre Aufgaben nach und nach an das BAWI, die NGO, die Privatwirtschaft und die betroffenen Universitäten übertragen wird. Stellen wir also diese nationale dreigliedrige Kommission unverzüglich auf. Versuchen wir dann sofort, in gleicher Weise für Vietnam vorzugehen, um die Zusammenarbeit, die sich mit diesem Land abzeichnet, mit den bestmöglichen Startchancen anzugehen. 